\title{
Aetiology, diagnosis, and clinical management of vulvodynia
}

\author{
Polina Vasileva ${ }^{1}$, Strahil A. Strashilov², Angel D. Yordanov ${ }^{3}$ \\ ${ }^{1}$ Department of Obstetrics and Gynaecology, Medical University of Pleven, Bulgaria \\ 2Department of Plastic Restorative, Reconstructive and Aesthetic Surgery, Medical University of Pleven, Bulgaria \\ ${ }^{3}$ Department of Gynaecological Oncology, Medical University of Pleven, Bulgaria
}

\begin{abstract}
Chronic vulvar pain or discomfort for which no obvious aetiology can be found, i.e. vulvodynia, can affect up to $16 \%$ of women, and it may be found in girls and women across all age groups and ethnicities. Most patients describe it as burning, stinging, irritation, or rawness. The symptoms may spread to the whole vulva (generalised vulvodynia) or only to part of it, such as the clitoris (clitorodynia) or the vestibule of the vagina (vestibulodynia). This condition is often underreported and underrecognised by health care providers. Vulvodynia is a significant burden to society, the health care system, the affected women, and their intimate partners. It has a negative impact on quality of life. Vulvodynia is a diagnosis of exclusion with unknown aetiology. The gynaecologist plays a key role in excluding other causes of vulvar pain, and collaborating with other health care providers to manage the patient's pain. Although many therapeutic options are available, such as vulvar care measures, psychological approaches, local treatment, oral medications, surgical procedures, electrical nerve stimulation, and laser therapy, there is no single treatment effective for all patients. That is why individualised management is needed. An individualised, holistic, and often multidisciplinary approach is needed to effectively manage the patient's pain and pain-related distress.
\end{abstract}

Key words: vulvodynia, aetiology, diagnosis, treatment.

\section{Introduction}

Many women feel vulvar pain or discomfort, which affects their quality of life. The vulvar pain may be caused by a specific disease or may be idiopathic. Idiopathic vulvar pain is classified as vulvodynia. Vulvodynia is defined as chronic pain or discomfort in the region of the vulva for more than three months, with no aetiological cause found [1]. If there is a present cause for the condition (lichen sclerosis, inflammation, etc.), this is not a case of vulvodynia. The symptoms may be described as itching, burning, stinging, irritation, stabbing, and/or rawness. The classification of vulvodynia is based on the description of the pain itself. The symptoms may involve the whole vulva (generalised vulvodynia) or may be localised in certain portions of the genitalia such as the clitoris (clitorodynia) or the vestibule of the vagina (vestibulodynia). Likewise, depending on whether there is a provoking aspect or not, the vulvodynia may be provoked vulvodynia (caused by placement of a swab, sexual intercourse), unprovoked vulvodynia (if there is no provoking aspect), or mixed. It is divided into primary and secondary depending on its occurrence. It also may be divided into intermittent, persistent, constant, immediate, or delayed [1].
Vulvodynia affects women of every age, reproductive period, and ethnicity. The lifetime prevalence of this condition has been estimated at $8 \%$, and this prevalence remains constant across all decades up to the age of 70 years. The average age of the onset of this condition is approximately 30 years, while it varies extensively in the range from 6 to 70 years of age [2]. Women presenting with vulvodynia are typically in stable, long-term relationships, they have had the pain for several years, and have been examined several times by multiple physicians before receiving the diagnosis [3].

Women with vulvodynia usually search for medical aid from different health care providers - family doctors, gynaecologists, urologists, dermatologists, and others, and, as is frequently the case, they are not familiar with the condition. This results in a delay of making a diagnosis and providing treatment. Even when the diagnosis is made, a major proportion of specialists face the challenge of the condition's therapy [4]. Vulvodynia has a significantly negative influence on the psycho-sexual condition of women and their quality of life. The chronic vulvar pain may result in frustration, chronic stress, and depression in women [5, 6]. Many women with vulvodynia feel pain with sexual inter- 
course, and the majority report difficulties in their sexual life [7]. Vulvodynia is a significant burden to society, health care systems, the affected women, their families, and/or their intimate partners in the same way as some more well-known diseases like urinary incontinence [8].

\section{Aetiology}

There are multiple theories for the occurrence of this condition and aetiology is still unknown. It is possible at a local level - to speak of a primary trigger causing inflammation or direct trauma of the vulva. This results in stimulation of pain receptors and possibly to receptor or nerve impairment. Contact allergy, vaginal fungal infection, or other urovaginal infections are taken into consideration as a cause of inflammation [9]. There are women who identify differently by a type trauma as the triggering aspect for their pain. Direct trauma of the vulva (e.g. episiotomy) or direct/indirect trauma of the pelvic fundus (if there is operative vaginal parturition) may also be mentioned as triggering factors. There are cases in which the onset of symptoms is connected with hormonal initiation (intake of birth control pills, menopause, and parturition). Chronic inflammation is frequently observed, which may lead to nociceptive pain as a result of peripheral sensibilisation, and, thereafter, to central sensibilisation - neuropathic pain. This sensibilisation is present and symptomatic for a long time after the primary trigger [9]. Possible contributing causes include injury or inflammation of the nerves, which transmit pain from the vulva to the spine, an increase of the number and sensitivity of nerve endings in the vulva, increased levels of inflammatory substances in the vulva, abnormal reaction to factors of the environment, genetical predisposition, weakness of the muscles of the pelvic fundus, spasm, or instability [10]. Women with vulvodynia are often very distressed about not being able to identify a "cause" for their pain. The aetiology of vulvodynia is uncertain and probably multifactorial.

\section{Clinical diagnosis}

The diagnosis of vulvodynia is made based on the principle of exclusion. In fact, the exclusion of all other treatable causes before making a diagnosis of vulvodynia is obligatory. A detailed medical history is necessary based on patient-reported written data, which includes the duration of pain syndrome, detailed history of the medical condition, sexual history, allergies, and previous treatment.

A good knowledge of vulvar anatomy is of exceptional significance for making of the diagnosis (Fig. 1). Cotton swab testing is used in order to identify the zones of pain (Fig. 2). It is also used in order to classify the pain as weak, moderate, or severe in all of the in- volved zones, and to carry out differentiation between localised and generalised forms of the condition. Very useful in cases with vulvodynia may be the use of a diagram of pain according to time. The vulva and vagina must be examined thoroughly. The presence of vulvovaginal infection must be ruled out, when it is necessary [11]. The use of vulvoscopy is not necessary, as well as the application of acetic acid 3-5\% [12], which may result in severe reaction on the part of the patient.

Typical physical finding upon the examination is erythema of vestibule. Since the diagnosis of vulvodynia is made based on the absence of any other cause of the condition, in the presence of erythema, vulvovaginal infection or other specific disease of the vulva is to be ruled out. Testing for human papilloma virus infection is unnecessary. The role of biopsy in making the diagnosis remains uncertain. Clinically significant dermatoses found by means of analysis of biopsic material from the vulva are found in, on average, 61\% (from 55\% to $90 \%$ of biopsic materials) of patients with refractory vulvodynia [13], i.e. although there is no particular his-

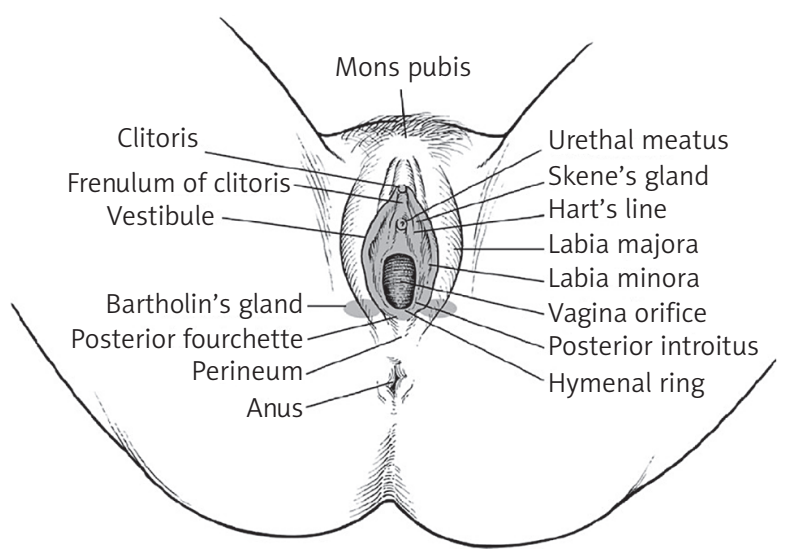

Fig. 1. Vulvar anatomy

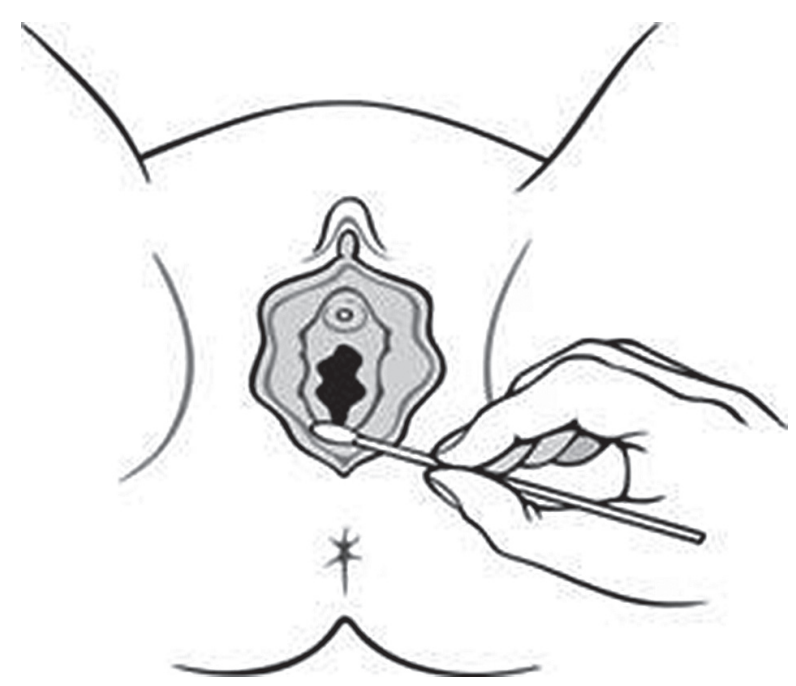

Fig. 2. Cotton swab test 
topathological finding for vulvodynia, the clinician has to assess the need for vulvar biopsy in order to exclude other aetiologies. The vulvar pain may also be reflected pain from other parts of the body, such as the back or flanks, so musculoskeletal evaluation should be considered [14]. The musculoskeletal assessment would help in ruling out of factors associated with vulvodynia such as pelvic muscular hyperactivity and myofascial or other biomechanical disorder.

\section{Treatment}

Vulvodynia is a complex disorder that can be difficult to treat. There are a lack of data about the effectiveness of various treatment options for vulvodynia, and many of the commonly recommended treatments have not been systematically studied in randomised, controlled trials. Nevertheless, several treatments have been used, with varying degrees of success. A review of treatment modalities proposed by most of the clinicians, involved in managing these patients, advocated initial utilisation of non-invasive therapies and then gradually proceeding to more aggressive therapies.

\section{Vulvar care measures}

Taking measures for delicate genital hygiene is necessary. Frequent recommendations concern the use of non-irritating, cotton underwear, avoiding of the use of vulvar irritating agents (perfumes, shampoos, showers), and use of soft, non-irritating soaps. It is possible for the vulva to be cleaned only with clean water and subsequent use of a moisture-preserving emollient in the involved area in order to prevent excessive drying and to act as a barrier function. During menstruation the usage of delicate cotton sanitary pads is strongly recommended. Good drying and cleaning of the vulva is necessary after urination. Good lubrication with non-irritating agents is recommendable for sexual intercourse [14].

\section{Psychological approaches}

Cognitive behavioural therapy is associated with a 30\% decrease in reported vulvar pain with intercourse [15]. Although there is no proven connection between vulvodynia and psychological predisposition, pain may be related with interpersonal or individual psychological problems. Marriage, sexual, or individual psychological consultation is also recommended in patients with similar symptoms.

\section{Physical therapy}

The physical therapy has also proven itself as a method of choice in connection with the combined treatment of vulvodynia [16]. It includes assessment of the patient's pelvic muscles, joints, fasciae, and ligaments. The assessment of the adjacent pelvic organs urinary bladder, large intestine - is also necessary. Most of the therapies include weekly sessions of one or two hours, which are focused most frequently on training of the muscles of the pelvic fundus, soft tissue mobilisation, joint manipulation, muscle relaxation, and general tone balance. Unfortunately, there is no standardised approach regarding effective physical therapy in connection with vulvodynia, and that is why the results cannot be accurately validated nor reproduced.

\section{Topical therapies}

Different topical medications have been tried as treatments for vulvar pain. In women who have been using multiple topical medications for a prolonged period, stopping all treatments may reduce symptoms [15].

Frequently used local medications include a variety of local anaesthetics, oestrogen creams, and tricyclic antidepressants in topical form. Although local steroids as a whole do not result in improvement in patients with vulvodynia, trigger-point injections of a combination of steroid and bupivacaine have been successful for some patients with localised vulvodynia [14].

The most commonly used local medication is lidocaine ointment $5 \%$, which is used 30 minutes before sex or when the patient has complaints. Male sexual partners may experience penile numbness and should avoid oral contact. Prolonged usage overnight of topical lidocaine can minimise feedback amplification of pain and may allow for healing [17]. Other studies report that lidocaine $5 \%$ cream was found to be less effective than topical placebo (20\% versus $33 \%$ response rate, respectively) [18]. Other topical agents found to be no more effective than placebo include cromolyn $4 \%$ and nifedipine [19]. Oestrogen has been used topically with variable results. For some patients with localised pain and vaginismus, a combination of topical amitriptyline $2 \%$ and baclofen $2 \%$ in a water-washable base has been useful for point tenderness and vaginismus. A compounding pharmacy is used to formulate these topical medications [14].

It is very important to choose the appropriate medication - for example ointments are usually better tolerated than creams because creams contain preservatives and stabilisers and often produce burning on application, whereas ointments are usually better tolerated [14].

\section{Oral medications}

A variety of oral medications are used for pain control. Because the pain of vulvodynia seems to be neuropathic, many medications that have been used effectively in the 
treatment of other neuropathic disorders have also been used in patients with vulvodynia. When a medication is added in the treatment of the condition, its prior check for drug interactions with the patient's current medication is necessary. Tricyclic antidepressants and anticonvulsants can also be used for vulvodynia pain control. When the therapy initiates, avoidance of polypharmacy is desirable. One drug should be prescribed at a time. Before initiation of therapy with antidepressants or anticonvulsants in patients with child-bearing potential, the need of anticonception has to be explained. Such medications are amitriptyline and gabapentin.

Other options are the use of calcium citrate combined with a low-oxalate diet, and the continuous treatment with an oral fluconazole in cases of proven candida infection. Narcotics and analgesics, such as non-steroidal anti-inflammatory drugs and acetaminophen, have not proven helpful.

\section{Electrical nerve stimulation}

An emerging treatment for vulvodynia is transcutaneous electrical nerve stimulation. Transcutaneous electrical nerve stimulation is effective in other chronic pain conditions. Improvement of pain syndrome and improvement of sexual function with its application was noted in women with vestibulodynia [20].

\section{Surgery}

When a series of non-surgical methods have been tried and failed, and the pain has been localised in the vestibule, vestibulectomy may be an effective treatment. It represents an excision in the region of the painful part of vestibule to the lateral vestibular walls at Hart's line, including all tender parts extending to the anterior vestibule, if necessary [21]. The success of vestibulectomy varies between 60 and $90 \%$ in comparison with the success of non-surgical methods - between $40 \%$ and $80 \%$.

In fact, there is a group of patients who would have better results with vestibulectomy. Patients with secondary dyspareunia have greater chances of improvement in comparison with patients with primary dyspareunia. Patients with dyspareunia in the context of permanent pain are less likely to be affected by means of surgical excision [11].

\section{Laser therapy}

Laser ablation of the vulvar epithelium with the KTP-Nd: YAG laser and the $\mathrm{CO}_{2}$ laser presents as an alternative to the more aggressive treatment by means of vestibulectomy. Based on the angiogenesis and increased nerve sensitivity, which characterise vulvodynia, the laser is used with the purpose of disturbance of these histological abnormalities, and to cause collagen remodelling, without altering the macroscopic anatomy. Results of laser therapy for vulvodynia are comparable to vestibulectomy. Complete response has been reported in $62 \%$ and improvement in $92 \%$ of patients [22].

\section{Applications of platelet-rich plasma}

This is a new, alternative method for treatment of some gynaecological disorders such as vulvar dystrophy, urogenital disorders, female sexual dysfunction, and others [23]. There is no scientific conformation of positive results of this treatment for vulvodynia, but it is a potential opportunity and the future will show if it can become a part of the treatment options.

\section{Complementary and alternative therapies for chronic vulvar pain}

Many women also use other types of therapy when they encounter the conventional diagnosis and treatment of their vulvar symptoms. The majority of women (approximately 88\%) had used at least one complementary health method, and $77 \%$ reported of the use of dietary supplements. This complementary health method and dietary supplements are differently maintained, and include a change in the dietary regimen, reduction of smoking and consumption of alcohol, a change in clothes, exercise, relaxation, massage, local application of ice, and saltwater baths [13]. Multiple products are used, which include products for skin care, skin antiseptics, anaesthetic creams, dietary and vegetal supplements, homeopathic treatment, antimycotic creams, etc. The overall success of self-diagnosis and treatment remains unclear [14].

\section{Conclusions}

Vulvodynia is a disease that is not well known in the circles of health care providers. The lack of clarity in its aetiology results in the presence of multiple methods for treatment, with various durations and levels of success. None of them can be effective in all cases. This requires use of a strictly individual approach to every single patient.

\section{Disclosure}

The authors report no conflict of interest. 


\section{References}

1. Haefner HK. Report of the International Society for the Study of Vulvovaginal Disease: terminology and classification of vulvodynia. J Low Genit Tract Dis 2007;11: 48-49.

2. Reed BD, Harlow SD, Sen A, et al. Prevalence and Demographic Characteristics of Vulvodynia in a Population-Based Sample. Am J Obstet Gynecol 2012; 206: 170.e1-e9.

3. Reed BD, Crawford S, Couper M, et al. Pain at the Vulvar Vestibule: a Web-Based Survey. J Low Genit Tract Dis 2004; 8: 48-57.

4. Sadownik LA. Clinical Profile of Vulvodynia Patients: a Prospective Study of 300 Patients. J Reprod Med 2000; 45: 679-684.

5. Nunns D, Mandal D. Psychological and Psychosexual Aspects of Vulvar Vestibulitis. Genitourin Med 1997; 73: 541-544.

6. Ehrstrom S, Kornfeld D, Rylander E, Bohm-Starke N. Chronic Stress in Women with Localised Provoked Vulvodynia. J Psychosom Obstet Gynaecol 2009; 30: 73-79.

7. Reed BD, Advincula AP, Fonde KR, et al. Sexual Activities and Attitudes of Women with Vulvar Dysesthesia. Obstet Gynecol 2003; 102: 325-331.

8. Kołodyńska G, Zalewski M, Rożek-Piechura K. Urinary incontinence in postmenopausal women - causes, symptoms, treatment. Prz Menopauzalny 2019; 18: 46-50.

9. Sadownik LA. Etiology, Diagnosis, and Clinical Management of Vulvodynia. Int J Womens Health 2014; 6: 437-449.

10. Bornstein J, Goldstein AT, Stockdale CK, et al. 2015 ISSVD, ISSWSH, and IPPS Consensus Terminology and Classification of Persistent Vulvar Pain and Vulvodynia. J Sex Med 2016; 13: 607-612.

11. Persistent Vulvar Pain. Committee Opinion No. 673. American College of Obstetricians and Gynecologists. Obstet Gynecol 2016; 128: e78-84.

12. Micheletti L, Bogliatto F, Lynch PJ. Vulvoscopy: Review of a Diagnostic Approach Requiring Clarification. J Reprod Med 2008; 53: 179-182.

13. Bowen AR, Vester A, Marsden L, et al. The Role of Vulvar Skin Biopsy in the Evaluation of Chronic Vulvar Pain. Am J Obstet Gynecol 2008; 199: 467.e1-6.

14. Haefner HK, Collins ME, Davis GD, et al. The Vulvodynia Guideline. J Low Genit Tract Dis 2005; 9: 40-51.

15. Bergeron S, Binik YM, Khalife S, et al. A Randomized Comparison of Group Cognitive-Behavioral Therapy, Surface Electromyographic Biofeedback, and Vestibulectomy in the Treatment of Dyspareunia Resulting from Vulvar Vestibulitis. Pain 2001; 91: 297-306.

16. Prendergast SA. Pelvic floor physical therapy for vulvodynia: a clinician's guide. Obstetr Gynecol Clin 2017; 44: 509-522.

17. Zolnoun DA, Hartmann KE, Steege JF. Overnight $5 \%$ lidocaine ointment for treatment of vulvar vestibulitis. Obstet Gynecol 2003; 102: 84-87.

18. Segal D, Tifheret H, Lazer S. Submucous Infiltration of Betamethasone and Lidocaine in the Treatment of Vulvar Vestibulitis. Eur J Obstet Gynecol Reprod Biol 2003; 107: 105-106.

19. Nyirjesy P, Sobel JD, Weitz MV, et al. Cromolyn cream for recalcitrant idiopathic vulvar vestibulitis: results of a placebo controlled study. Sex Transm Infect 2001; 77: 53-57.

20. Murina F, Bianco V, Radici G, et al. Transcutaneous Electrical Nerve Stimulation to Treat Vestibulodynia: a Randomised Controlled Trial. BJOG 2008; 115: 1165-1170.

21. Tommola P, Unkila-Kallio L, Paavonen J. Surgical Treatment of Vulvar Vestibulitis: a Review. Acta Obstet Gynecol Scand 2010; 89: 1385-1395.

22. Bergeron S, Binik YM, Khalife S, Pagidas K. Vulvar Vestibulitis Syndrome: a Critical Review. Clin J Pain 1997; 13: 27-42.

23. Dawood AS, Salem HA. Current clinical applications of platelet-rich plasma in various gynecological disorders: An appraisal of theory and practice. Clin Exp Reprod Med 2018; 45: 67-74. 